\title{
Long-Term Low Intensity Physical Exercise Attenuates Heart Failure Development in Aging Spontaneously Hypertensive Rats
}

\author{
Luana U. Pagan ${ }^{a}$ Ricardo L. Damatto ${ }^{a}$ Marcelo D.M. Cezar ${ }^{a}$ Aline R.R. Lima ${ }^{a}$ \\ Camila Bonomo ${ }^{a}$ Dijon H.S. Campos ${ }^{a}$ Mariana J. Gomes ${ }^{a}$ Paula F. Martinez ${ }^{b}$ \\ Silvio A. Oliveira Jrb Rodrigo Gimenes ${ }^{a}$ Camila M. Rosa ${ }^{a}$ Daniele M. Guizonia \\ Yasmin C. Moukbela Antonio C. Cicogna ${ }^{a}$ Marina P. Okoshi ${ }^{a}$ Katashi Okoshia \\ aInternal Medicine Department, Botucatu Medical School, Univ. Estadual Paulista, UNESP, Botucatu, \\ Sao Paulo, 'bederal University of Mato Grosso do Sul, Campo Grande, Brazil
}

\section{Key Words}

Spontaneously hypertensive rat - Tissue Doppler imaging • Echocardiography • Papillary muscle $\cdot$ Myocardial fibrosis

\begin{abstract}
Background: Physical exercise is a strategy to control hypertension and attenuate pressure overload-induced cardiac remodeling. The influence of exercise on cardiac remodeling during uncontrolled hypertension is not established. We evaluated the effects of a long-term low intensity aerobic exercise protocol on heart failure (HF) development and cardiac remodeling in aging spontaneously hypertensive rats (SHR). Methods: Sixteen month old SHR $(n=50)$ and normotensive Wistar-Kyoto $(\mathrm{WKY}, \mathrm{n}=35)$ rats were divided into sedentary (SED) and exercised (EX) groups. Rats exercised in treadmill at $12 \mathrm{~m} / \mathrm{min}, 30 \mathrm{~min} /$ day, 5 days/week, for four months. The frequency of HF features was evaluated at euthanasia. Statistical analyses: ANOVA and Tukey or Mann-Whitney, and Goodman test. Results: Despite slightly higher systolic blood pressure, SHR-EX had better functional capacity and lower HF frequency than SHR-SED. Echocardiography and tissue Doppler imaging showed no differences between SHR groups. In SHR-EX, however, left ventricular (LV) systolic diameter, larger in SHR-SED than WKY-SED, and endocardial fractional shortening, lower in SHR-SED than WKY-SED, had values between those in WKY-EX and SHR-SED not differing from either group. Myocardial function, assessed in LV papillary muscles, showed improvement in SHR-EX over SHR-SED and WKY-EX. LV myocardial collagen fraction and type I and III collagen gene expression were increased in SHR groups. Myocardial hydroxyproline concentration was lower in SHR-EX than SHR-SED. Lysyl oxidase gene expression was higher in SHR-SED than WKY-SED. Conclusion: Exercise improves functional capacity and reduces decompensated HF in aging SHR independent of elevated arterial pressure. Improvement in functional status is combined with attenuation of LV and myocardial dysfunction and fibrosis.
\end{abstract}

Copyright (C) 2015 S. Karger AG, Basel

Katashi Okoshi

KARGER 125
Departmento de Clinica Medica, Faculdade de Medicina de Botucatu, UNESP Rubiao Junior, S/N, CEP 18618970 Botucatu, SP (Brazil)

Tel. +55 14 3880-1171, Fax +55 143882 2238, E-Mail katashi@fmb.unesp.br 


\section{Cellular Physiology Cell Physiol Biochem 2015;36:61-74 \begin{tabular}{l|l} 
and Biochemistry Published ontIne: AprII 27, 2015 & $\begin{array}{l}\text { C 2015 S. Karger AG, Basel } \\
\text { www.karger.com/cpb }\end{array}$ \\
\hline
\end{tabular}}

Pagan et al.: Exercise in Aging Spontaneously Hypertensive Rats

\section{Introduction}

Systemic arterial hypertension is a major public health problem due to its high prevalence and a large percentage of uncontrolled patients in the general population, despite the widespread availability of anti-hypertensive drugs [1]. Long-term untreated hypertension is a major cause of cardiac remodeling which evolves progressively from compensatory left ventricular hypertrophy to left ventricular dysfunction and heart failure [2, 3].

The transition from compensated cardiac hypertrophy to decompensated heart failure is characterized by several myocardial alterations such as cardiomyocyte loss and dysfunction, interstitial fibrosis, changes in intracellular calcium transients, fetal gene reprogramming, and inflammatory activation $[2,4,5]$. Interstitial matrix is a key component involved in heart failure development $[6,7]$. Experimental studies on spontaneously hypertensive rats have suggested that fibrosis and underlying connective tissue response events are important in the transition from compensated hypertrophy to failure [6].

Physical exercise is an established non-pharmacological therapeutic adjuvant strategy in controlling hypertension and preventing or attenuating chronic pressure overload-induced cardiac remodeling $[8,9]$. In stable chronic heart failure, clinical studies have shown that long-term moderate physical training attenuates abnormal cardiac remodeling and improves functional capacity and quality of life [10-13]. In different cardiac injury models, exercise has been shown to attenuate left ventricular dilation, myocyte hypertrophy, myocardial fibrosis, mitochondrial dysfunction, myocyte calcium handling changes, sympathoexcitation, cardiac dysfunction, and improve inflammatory profile [14-22].

However, the effects of an exercise program on cardiac remodeling during uncontrolled hypertension have not been established. The spontaneously hypertensive rat (SHR) is a wellestablished model of genetic hypertension and hypertensive cardiomyopathy [23, 24]. At one month old, arterial hypertension starts to increase stimulating left ventricular hypertrophy $[25,26]$. If pressure overload is sustained, cardiac decompensation ensues, usually at 18-22 months of age [23, 24, 27]. In SHR, Schultz et al. [28] observed that long-term (from 6 to 22 months of age) voluntary wheel running was associated with impaired cardiac remodeling shown by increased left ventricular dilation, myocardial collagen content, myocyte crosssectional area and volume, and reduced systolic function. Voluntary wheel running is characterized as a series of short relatively high-intensity bouts [29]. Therefore, whether lighter aerobic exercise protocols can improve cardiac remodeling and function in SHR is uncertain. Thus, the aim of this study was to evaluate the influence of a long-term low intensity aerobic exercise protocol on heart failure development, cardiac remodeling, and left ventricular and myocardial function in aging SHR.

\section{Material and Methods}

Experimental groups

Sixteen-month-old male SHR and normotensive Wistar-Kyoto (WKY) rats were purchased from the Central Animal House at Botucatu Medical School, UNESP. All animals were housed in a room under temperature control at $23{ }^{\circ} \mathrm{C}$ and kept on a 12-hour light/dark cycle. Food and water were supplied ad libitum. All experiments and procedures were approved by Botucatu Medical School Ethics Committee, Botucatu, SP, Brazil.

Rats were assigned to four groups: sedentary WKY group (WKY-SED, $n=17$ ); exercised WKY group (WKY-EX, $n=18$ ); sedentary SHR group (SHR-SED, $n=25$ ); exercised SHR group (SHR-EX, $n=25)$. The physical exercise protocol was started at the age of 16 months and applied for four months. The exercise protocol consisted of $30 \mathrm{~min} /$ day treadmill running 5 days/week. During an adaptive period, exercise velocity was slowly increased from $5 \mathrm{~m} / \mathrm{min}$ to $12 \mathrm{~m} / \mathrm{min}$, and exercise duration from 10 to $30 \mathrm{~min}$ [30, 31]. In the first two weeks of training, the animals were subjected to low-voltage electrical stimulation to start exercise. No animals were lost during exercise training.

Systolic arterial pressure, physical capacity, cardiac structures, and left ventricular function were assessed before and after the exercise period. Systolic arterial pressure was measured by pletismography 


\section{Cellular Physiology Cell Physiol Biochem 2015;36:61-74 \begin{tabular}{l|l} 
and Biochemistry Publisned ontIne: AprII Z7, 2015 & $\begin{array}{l}\text { C 2015 S. Karger AG, Basel } \\
\text { www.karger.com/cpb }\end{array}$ \\
\hline
\end{tabular}}

Pagan et al.: Exercise in Aging Spontaneously Hypertensive Rats

using the tail-cuff method (Narco Bio-System ${ }^{\circledR}$, model 709-0610, International Biomedical, Inc, USA). At euthanasia two observers determined the presence or absence of clinical and pathological congestive heart failure features. The clinical finding suggestive of heart failure was tachypnea/labored respiration. Pathologic assessment of cardiac decompensation included subjective evaluation of pleuropericardial effusion, atrial thrombi, ascites, and liver congestion. Lung congestion and right ventricular hypertrophy were determined according to their normalized weight. Rats were considered to have pulmonary congestion when lungs weight-to-body weight ratio > 2 standard deviations above the mean for the WKY-SED group, and right ventricular hypertrophy when right ventricle weight-to-body weight ratio $>0.8 \mathrm{mg} / \mathrm{g}[32,33]$.

\section{Maximal exercise capacity}

Before evaluating maximal exercise capacity, the rats were adapted to treadmill exercise over 5 days (10 min/day). Exercise testing was performed on a graded treadmill. The speed started at $6 \mathrm{~m} / \mathrm{min}$ and was increased by $3 \mathrm{~m} / \mathrm{min}$ every $3 \mathrm{~min}$ until rats were unable to run [19]. Rats were considered to be exhausted when they refused to run even after sound stimulation or were unable to coordinate steps. Maximum velocity was recorded and total distance calculated.

\section{Echocardiographic study}

Echocardiographic evaluation was performed using a commercially available echocardiograph (General Electric Medical Systems, Vivid S6, Tirat Carmel, Israel) equipped with a 5 - $11.5 \mathrm{MHz}$ multifrequency probe. Rats were anesthetized by intramuscular injection of a mixture of ketamine (50 mg/ $\mathrm{kg}$ ) and xylazine $(0.5 \mathrm{mg} / \mathrm{kg})$. A two-dimensional parasternal short-axis view of the left ventricle (LV) was obtained at the level of the papillary muscles. M-mode tracings were obtained from short-axis views of the $\mathrm{LV}$ at or just below the tip of the mitral-valve leaflets, and at the level of the aortic valve and left atrium [3436]. M-mode images of the LV were printed on a black-and-white thermal printer (Sony UP-890MD) at a sweep speed of $100 \mathrm{~mm} / \mathrm{s}$. All LV structures were manually measured by the same observer (KO) using the leading-edge method of the American Society of Echocardiography [37]. Measurements were the mean of at least five cardiac cycles on M-mode tracings. The following structural variables were measured: left atrium (LA) diameter, LV diastolic and systolic dimensions (LVDD and LVSD, respectively), LV diastolic posterior wall thickness (PWT), LV diastolic septal wall thickness (SWT), and aortic diameter (AO). Left ventricular mass (LVM) was calculated using the formula [(LVDD + PWT $\left.+\mathrm{SWT}^{3}-\mathrm{LVDD}^{3}\right] \times 1,04 . \mathrm{LV}$ relative wall thickness (RWT) was calculated by the formula $2 \times$ PWT/LVDD. LV function was assessed by the following parameters: endocardial fractional shortening (EFS), midwall fractional shortening (MFS), ejection fraction (EF), posterior wall shortening velocity (PWSV), early and late diastolic mitral inflow velocities (E and A waves), E/A ratio, and isovolumetric relaxation time (IVRT). A joint assessment of diastolic and systolic LV function was performed by the myocardial performance index (Tei index). The study was complemented with evaluation by tissue Doppler imaging (TDI) of systolic ( $\left.S^{\prime}\right)$, early diastolic ( $\left.E^{\prime}\right)$, and late ( $\left.A^{\prime}\right)$ velocity of the mitral annulus (arithmetic average travel speeds of the lateral and septal walls), and E'/A' and E/E' ratios.

\section{Myocardial functional study}

Two days after the final echocardiographic study, myocardial intrinsic contractile performance was evaluated in isolated LV papillary muscle preparation as previously described [38-40]. Rats were anesthetized (pentobarbital sodium, $50 \mathrm{mg} / \mathrm{kg}$, intraperitoneal) and decapitated. Hearts were quickly removed and placed in oxygenated Krebs-Henseleit solution at $28{ }^{\circ} \mathrm{C}$. $\mathrm{LV}$ anterior or posterior papillary muscle was dissected free, mounted between two spring clips, and placed vertically in a chamber containing Krebs-Henseleit solution at $28{ }^{\circ} \mathrm{C}$ and oxygenated with a mixture of $95 \% \mathrm{O}_{2}$ and $5 \% \mathrm{CO}_{2}(\mathrm{pH} 7.38$ ). The composition of the Krebs-Henseleit solution in $\mathrm{mM}$ was as follows: $118.5 \mathrm{NaCl}, 4.69 \mathrm{KCl}, 1.25 \mathrm{CaCl}_{2}, 1.16$ $\mathrm{MgSO}_{4}, 1.18 \mathrm{KH}_{2} \mathrm{PO}_{4}, 5.50$ glucose, and $25.88 \mathrm{NaHCO}_{3}$. The spring clips were attached to a Kyowa model 120T-20B force transducer and a lever system, which allowed for muscle length adjustment. Preparations were stimulated 12 times/min by parallel platinum electrodes delivering 5-ms pulses at a voltage $10 \%$ above threshold during equilibrium period and isometric contractions. After a 60 min period, during which the preparations were permitted to shorten while carrying light loads, muscles were loaded to contract isometrically and stretched to the apices of their length-tension curves (Lmax). After a 5 min period, during which preparations performed isotonic contractions, muscles were again placed under isometric conditions, 


\section{Cellular Physiology Cell Physiol Biochem 2015;36:61-74 \begin{tabular}{l|l} 
and Biochemistry Published onlIne: April 27, 2015 & $\begin{array}{l}\text { C 2015 S. Karger AG, Basel } \\
\text { www.karger.com/cpb }\end{array}$ \\
\hline
\end{tabular}}

Pagan et al.: Exercise in Aging Spontaneously Hypertensive Rats

and the apex of the length-tension curve was determined. A 15 min period of stable isometric contraction was imposed prior to the experimental period. One isometric contraction was then recorded for later analysis. The following parameters were measured from isometric contraction: peak of developed tension (DT, g/mm2), resting tension (RT, g/mm2), and maximum rate of tension development ( $+\mathrm{dT} / \mathrm{dt}, \mathrm{g} / \mathrm{mm}^{2} / \mathrm{s}$ ). To evaluate myocardial contractile reserve, papillary muscle mechanical performance was evaluated at basal condition and after the following positive inotropic stimulation: post-rest contractions $(10,30$, and $60 \mathrm{~s}$ ), extracellular $\mathrm{Ca}^{2+}$ concentration increase (external calcium concentrations of $0.625,1.25$, and 2.5 $\mathrm{mM})$, and $\beta$-adrenergic agonist isoproterenol $\left(10^{-8}, 10^{-7}\right.$, and $\left.10^{-6} \mathrm{M}\right)$ addition to the nutrient solution [41]. Papillary muscle cross-sectional area was calculated from muscle weight and length by assuming cylindrical uniformity and a specific gravity of 1.0. All force data were normalized for muscle cross-sectional area.

After dissecting papillary muscle, the ventricles, lungs, and atria were dissected and weighed. The tibia was removed and dried and its length measured. Left and right ventricular wet weight normalized by body weight and tibia length was used as indexes of ventricular hypertrophy. LV samples were immediately frozen in liquid nitrogen and stored at $-80^{\circ} \mathrm{C}$. Lungs were weighed before and after drying sessions $\left(65^{\circ} \mathrm{C}\right.$ for $72 \mathrm{~h}$ ) to evaluate wet/dry weight ratio.

\section{Morphologic study}

Frozen LV samples were transferred to a cryostat and cooled to $-20^{\circ} \mathrm{C}$. Serial transverse $8 \mu \mathrm{m}$ thick sections were stained with hematoxylin and eosin. At least 50 cardiomyocyte diameters were measured from each $L V$ as the shortest distance between borders drawn across the nucleus [41]. Other slides were stained with Sirius red F3BA and used to quantify interstitial collagen fraction [42]. On average, 20 microscopic fields were analyzed with a 40X lens. Perivascular collagen was excluded from this analysis. Measurements were taking using a compound microscope (Leica DM LS; Nussloch, Germany) attached to a computerized imaging analysis system (Media Cybernetics, Silver Spring, MD, USA).

\section{Myocardial hydroxyproline measurement}

Myocardial hydroxyproline concentration was estimated using a colorimetric assay (QuickZyme Hydroxyproline Assay, Leiden, Netherlands) according to manufacturer instructions.

\section{Real time RT-PCR analysis}

Total RNA was extracted from the LV with TRIzol Reagent (Invitrogen Life Technologies, Carlsbad, CA, USA) according to a previously described method [43-45]. Frozen muscles were mechanically homogenized on ice in $1 \mathrm{ml}$ of ice-cold TRIzol reagent. Total RNA was solubilized in RNase-free $\mathrm{H}_{2} \mathrm{O}$, incubated in DNase I (Invitrogen Life Technologies) to remove any DNA in the sample, and quantified by measuring optical density (OD) at $260 \mathrm{~nm}$. RNA purity was ensured by obtaining a $260 / 280 \mathrm{~nm}$ OD ratio of approximately 2.0 . One microgram of RNA was reverse transcribed using High Capacity cDNA Reverse Transcription Kit in a total volume of $20 \mu \mathrm{L}$, according to standard methods (Applied Biosystems, Foster City, CA, EUA). Aliquots of 2.5 $\mu \mathrm{L}$ (10-100 ng) of cDNA were then submitted to real-time PCR reaction using $10 \mu \mathrm{L} 2 \mathrm{X}$ TaqMan ${ }^{\circledR}$ Universal PCR Master Mix (Applied Biosystems) and $1 \mu \mathrm{L}$ of customized assay (20X) containing sense and antisense primers and Taqman (Applied Biosystems, Foster City, CA, EUA) probe specific to each gene, collagen type I (Taqman assay Rn00567418_m1; Ref. seq. Genbank NM_053304.1), collagen type III (Taqman assay Rn00598571_m1; Ref. seq. Genbank NM_032085.1), and lysyl oxidase (Taqman assay Rn00565920_m1; Ref. seq. Genbank NM_017061.2). Amplification and analysis were performed using Step One PlusTM Real Time PCR System (Applied Biosystems, Foster City, CA, EUA) according to manufacturer recommendations. Expression data were normalized to cyclofilin (reference gene; Taqman assay Rn00667869_m1; Ref. seq. Genbank NM_017101) expression. Reactions were performed in triplicate and expression levels calculated using the CT comparative method $\left(2^{-\Delta \Delta C T}\right)$.

\section{Statistical analysis}

Results are expressed in descriptive measurements. Variables were compared by two-factor ANOVA, followed, respectively, by the Tukey test, for parametric distributions, and the Dunn test, for non-parametric data. Frequency of heart failure features was assessed by the Goodman test. Statistical significance was accepted at $\mathrm{p}<0.05$.

\section{KARGER}




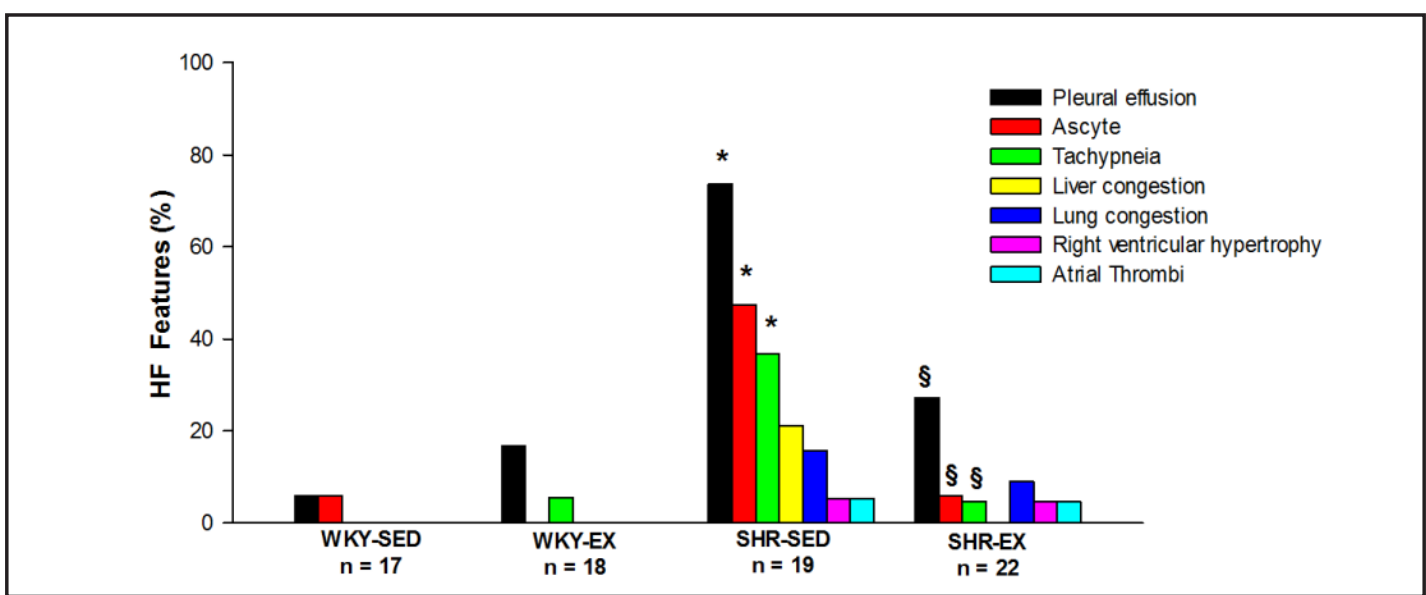

Fig. 1. Heart failure (HF) feature frequencies. WKY-SED: sedentary Wistar-Kyoto rats; WKY-EX: exercised Wistar-Kyoto rats; SHR-SED: sedentary spontaneously hypertensive rats; SHR-EX: exercised spontaneously hypertensive rats; Goodman test; * $\mathrm{p}<0.05$ vs WKY-SED; $§ \mathrm{p}<0.05$ vs SHR-SED.

Table 1. Systolic blood pressure and anatomical data. Data as mean \pm standard deviation. WKY-SED: sedentary Wistar-Kyoto rats; WKYEX: exercised Wistar-Kyoto rats; SHR-SED: sedentary spontaneously hypertensive rats; SHR-EX: exercised spontaneously hypertensive rats; SBP: systolic blood pressure; BW: body weight; LV: left ventricle weight; RV: right ventricle weight. ANOVA and Tukey or Dunn test; * $\mathrm{p}<0.05$ vs WKY-SED; \# $\mathrm{p}<0.05$ vs WKY-EX; § $\mathrm{p}<0.05$ vs SHR-SED

\begin{tabular}{lcccc}
\hline \multicolumn{1}{c}{ Variáveis } & $\begin{array}{c}\text { WKY-SED } \\
(\mathrm{n}=17)\end{array}$ & $\begin{array}{c}\text { WKY-EX } \\
(\mathrm{n}=18)\end{array}$ & $\begin{array}{c}\text { SHR-SED } \\
(\mathrm{n}=19)\end{array}$ & $\begin{array}{c}\text { SHR-EX } \\
(\mathrm{n}=19)\end{array}$ \\
\hline Initial SBP (mmHg) & $118 \pm 7.73$ & $122 \pm 5.10$ & $195 \pm 16.6^{*}$ & $194 \pm 18.6^{\#}$ \\
Final SBP (mmHg) & $127 \pm 24.8$ & $134 \pm 15.3$ & $212 \pm 22.4^{*}$ & $225 \pm 20.3^{\#}$ \\
Initial BW (g) & $619 \pm 65.2$ & $644 \pm 46.8$ & $401 \pm 24.1^{*}$ & $388 \pm 25.9^{\#}$ \\
Final BW (g) & $623 \pm 102$ & $612 \pm 81.5$ & $390 \pm 22.8^{*}$ & $371 \pm 28.4^{\#}$ \\
Tibia length (cm) & $4.76 \pm 0.14$ & $4.79 \pm 0.06$ & $4.17 \pm 0.05^{*}$ & $4.15 \pm 0.03^{\#}$ \\
LV (g) & $0.95 \pm 0.07$ & $1.03 \pm 0.08^{*}$ & $1.19 \pm 0.07^{*}$ & $1.17 \pm 0.13^{\#}$ \\
LV/BW (g/kg) & $1.53 \pm 0.14$ & $1.70 \pm 0.18^{*}$ & $3.09 \pm 0.22^{*}$ & $3.14 \pm 0.24^{\#}$ \\
LV/tibia length (g/cm) & $0.20 \pm 0.02$ & $0.22 \pm 0.02^{*}$ & $0.29 \pm 0.01^{*}$ & $0.28 \pm 0.03^{\#}$ \\
RV (g) & $0.27 \pm 0.02$ & $0.29 \pm 0.03^{*}$ & $0.24 \pm 0.03^{*}$ & $0.23 \pm 0.02^{\#}$ \\
RV/BW (g/kg) & $0.43 \pm 0.05$ & $0.47 \pm 0.06^{*}$ & $0.62 \pm 0.05^{*}$ & $0.61 \pm 0.06^{\#}$ \\
Atria (g) & $0.13 \pm 0.02$ & $0.14 \pm 0.02$ & $0.11 \pm 0.02^{*}$ & $0.11 \pm 0.01^{\#}$ \\
Atria/BW (g/kg) & $0.21 \pm 0.04$ & $0.23 \pm 0.04$ & $0.27 \pm 0.06^{*}$ & $0.29 \pm 0.04^{\#}$ \\
Lung wet/dry weight ratio & $4.47 \pm 0.18$ & $4.46 \pm 0.22$ & $5.28 \pm 0.47^{*}$ & $5.00 \pm 0.44^{\# \S}$ \\
Lung (g) & $2.81 \pm 0.44$ & $2.99 \pm 0.50$ & $3.06 \pm 0.56$ & $2.76 \pm 0.45$ \\
Lung/BW (g/kg) & $4.47 \pm 0.79$ & $4.97 \pm 1.07$ & $7.40 \pm 1.07^{*}$ & $7.09 \pm 1.05^{\#}$ \\
\hline
\end{tabular}

\section{Results}

Characterization of experimental groups and anatomic variables

During the experiment, 6 SHR-SED and 3 SHR-EX died; no rat from WKY groups died. Figure 1 shows the frequency of heart failure features. The SHR-SED had a higher frequency of pleural effusion, ascites, and tachypnea than WKY-SED and SHR-EX.

Systolic blood pressure and anatomical data are shown in Table 1. Blood pressure was higher in SHR than WKY before and after the exercise protocol and higher in SHR-EX than SHR-SED after training. Initial and final body weight (BW), tibia length, right ventricle weight (RV) and atria weight were decreased in SHR groups compared to their respective controls. $\mathrm{LV}, \mathrm{LV} / \mathrm{BW}, \mathrm{LV} /$ tibia length, $\mathrm{RV} / \mathrm{BW}$, atria/BW and wet/dry lung weight ratios were higher in SHR groups compared to controls. WKY-EX had higher values of LV, LV/BW, LV/tibia length, RV and RV/BW than WKY-SED.

\section{Exercise tolerance testing}

Functional capacity was evaluated as maximal exercise performance in running test until exhaustion. SHR groups presented better performance in the initial and final test than their respective controls. The exercise protocol increased run time and distance in both exercise compared to sedentary groups (Fig. 2). 


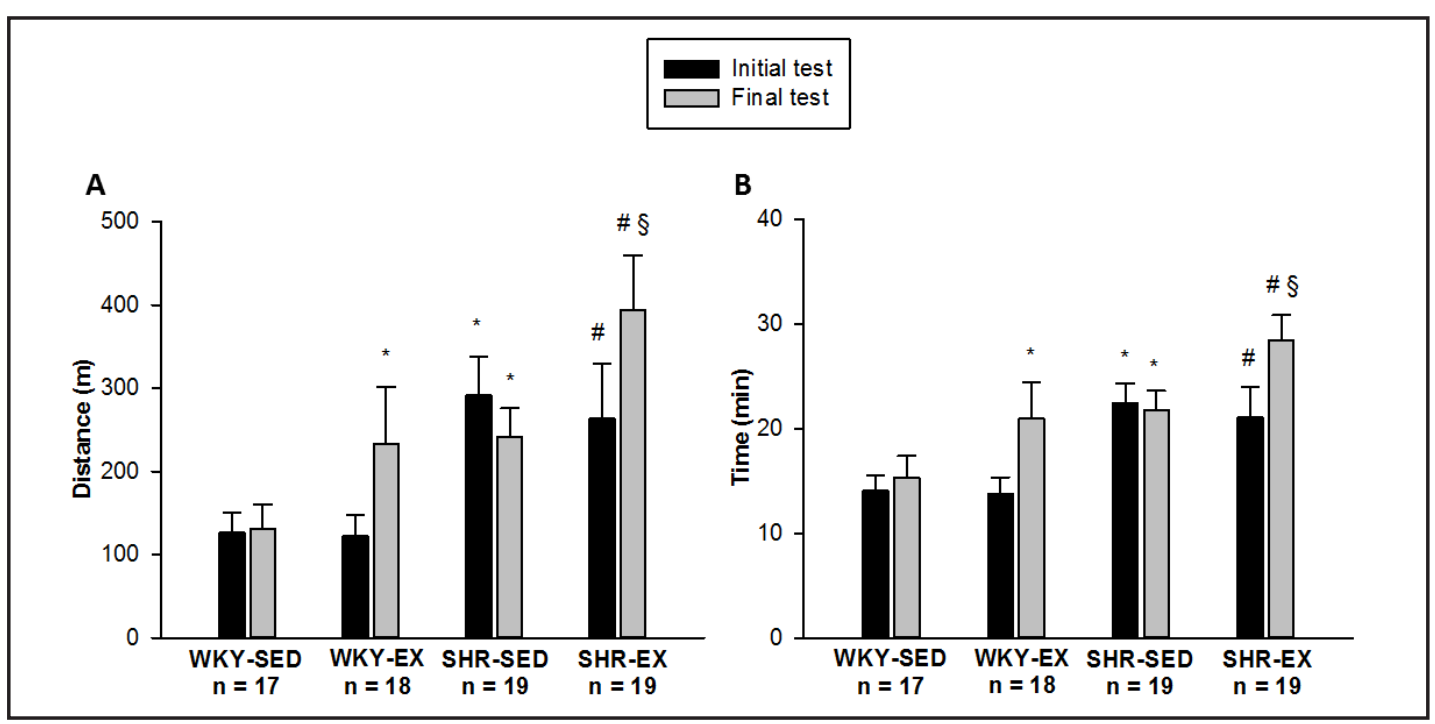

Fig. 2. Distance (A) and time (B) evaluated in treadmill running test until exhaustion. WKY-SED: sedentary Wistar-Kyoto rats; WKY-EX: exercised Wistar-Kyoto rats; SHR-SED: sedentary spontaneously hypertensive rats; SHR-EX: exercised spontaneously hypertensive rats. ANOVA and Tukey test; mean \pm standard deviation; * $\mathrm{p}<0.05$ vs WKY-SED; \# $\mathrm{p}<0.05$ vs WKY-EX; $§ \mathrm{p}<0.05$ vs SHR-SED.

Table 2. Echocardiographic structural data. Data as mean \pm standard deviation. WKY-SED: sedentary Wistar-Kyoto rats; WKY-EX: exercised WistarKyoto rats; SHR-SED: sedentary spontaneously hypertensive rats; SHR-EX: exercised spontaneously hypertensive rats; HR: heart rate; LVDD and LVSD: left ventricular (LV) diastolic and systolic diameter, respectively; BW: body weight; PWT: LV posterior wall thickness; SWT: LV septal wall thickness; AO: aorta diameter; LA: left atrial diameter; LVM: LV

\begin{tabular}{lcccc}
\hline & $\begin{array}{c}\text { WKY-SED } \\
(\mathrm{n}=17)\end{array}$ & $\begin{array}{c}\text { WKY-EX } \\
(\mathrm{n}=15)\end{array}$ & $\begin{array}{c}\text { SHR-SED } \\
(\mathrm{n}=13)\end{array}$ & $\begin{array}{c}\text { SHR-EX } \\
(\mathrm{n}=16)\end{array}$ \\
\hline HR $(\mathrm{bpm})$ & $270 \pm 71$ & $278 \pm 42$ & $302 \pm 47$ & $282 \pm 28$ \\
LVDD $(\mathrm{mm})$ & $8.19 \pm 0.61$ & $8.49 \pm 0.87$ & $8.72 \pm 0.77$ & $8.30 \pm 0.66$ \\
LVDD $/ \mathrm{BW}(\mathrm{mm} / \mathrm{kg})$ & $13.8 \pm 2.30$ & $14.4 \pm 2.85$ & $21.9 \pm 2.32^{*}$ & $22.2 \pm 2.23^{\#}$ \\
LVSD $(\mathrm{mm})$ & $3.42 \pm 0.84$ & $3.87 \pm 0.85$ & $4.26 \pm 0.91^{*}$ & $3.99 \pm 0.76$ \\
PWT $(\mathrm{mm})$ & $1.58 \pm 0.11$ & $1.58 \pm 0.10$ & $1.87 \pm 0.10^{*}$ & $1.88 \pm 0.20^{\#}$ \\
SWT $(\mathrm{mm})$ & $1.58 \pm 0.12$ & $1.59 \pm 0.10$ & $1.88 \pm 0.19^{*}$ & $1.88 \pm 0.19^{\#}$ \\
AO $(\mathrm{mm})$ & $4.34 \pm 0.26$ & $4.37 \pm 0.24$ & $4.87 \pm 0.21^{*}$ & $4.79 \pm 0.32^{\#}$ \\
LA $(\mathrm{mm})$ & $5.76 \pm 0.80$ & $6.14 \pm 0.94$ & $7.26 \pm 0.91^{*}$ & $7.04 \pm 0.91^{\#}$ \\
LA/AO & $1.33 \pm 0.19$ & $1.41 \pm 0.22$ & $1.50 \pm 0.23^{*}$ & $1.47 \pm 0.19$ \\
LA/BW (mm/kg) & $9.78 \pm 2.38$ & $10.38 \pm 2.02$ & $18.2 \pm 2.06^{*}$ & $18.8 \pm 2.47^{\#}$ \\
LVM $(\mathrm{g})$ & $0.96 \pm 0.17$ & $1.03 \pm 0.25$ & $1.34 \pm 0.23^{*}$ & $1.24 \pm 0.26^{\#}$ \\
LVMI $(\mathrm{g} / \mathrm{kg})$ & $1.60 \pm 0.31$ & $1.74 \pm 0.47$ & $3.37 \pm 0.65^{*}$ & $3.30 \pm 0.59^{\#}$ \\
RWT & $0.39 \pm 0.03$ & $0.37 \pm 0.03$ & $0.42 \pm 0.03^{*}$ & $0.44 \pm 0.04^{\#}$ \\
\hline
\end{tabular}
mass; LVMI: LV mass index; RWT: relative wall thickness. ANOVA and Tukey or Dunn test; * p<0.05 vs WKYSED; \# p<0.05 vs WKY-EX

\section{Echocardiographic analysis}

Echocardiography evaluation was performed before the exercise protocol to ensure homogeneity between groups (data not shown). At the end of the study, both SHR-SED and SHR-EX presented increased LV diastolic diameter/body weight ratio, LV posterior and septal wall thickness, left atrial diameter, LV mass, and relative wall thickness than their respective controls. SHR-EX LV systolic diameter was between that in WKY-EX and SHR-SED and did not significantly differ from either group (Table 2).

LV functional data are shown in Table 3. SHR-SED and SHR-EX had lower midwall fractional shortening and posterior wall shortening velocity, and a higher Tei index than their respective controls. Endocardial fractional shortening was lower in SHR-SED than WKY-SED; in SHR-EX, this parameter was not significantly different from WKY-EX and SHRSED. Isovolumic relaxation time was higher in SHR than WKY. The other parameters did not differ between groups. 
Pagan et al.: Exercise in Aging Spontaneously Hypertensive Rats

Table 3. Echocardiographic evaluation of left ventricle function. Data as mean \pm standard deviation. WKY-SED: sedentary Wistar-Kyoto rats; WKYEX: exercised Wistar-Kyoto rats; SHR-SED: sedentary spontaneously hypertensive rats; SHR-EX: exercised spontaneously hypertensive rats; EFS: endocardial fractional shortening; MFS: midwall fractional shortening; PWSV: posterior wall shortening velocity; TDI-S': mitral annular systolic velocity by tissue Doppler

\begin{tabular}{lcccc}
\hline & $\begin{array}{c}\text { WKY-SED } \\
(\mathrm{n}=17)\end{array}$ & $\begin{array}{c}\text { WKY-EX } \\
(\mathrm{n}=15)\end{array}$ & $\begin{array}{c}\text { SHR-SED } \\
(\mathrm{n}=13)\end{array}$ & $\begin{array}{c}\text { SHR-EX } \\
(\mathrm{n}=16)\end{array}$ \\
\hline EFS \% & $58.6 \pm 8.20$ & $54.8 \pm 5.86$ & $51.5 \pm 7.67^{*}$ & $52.2 \pm 6.13$ \\
MFS \% & $29.8 \pm 5.05$ & $28.0 \pm 5.05$ & $25.2 \pm 4.60^{*}$ & $23.9 \pm 3.71^{\#}$ \\
PWSV (mm/s) & $39.3 \pm 4.80$ & $37.5 \pm 5.54$ & $35.3 \pm 5,41^{*}$ & $34.4 \pm 4,04^{*}$ \\
Tei index & $0.50 \pm 0.06$ & $0.53 \pm 0.05$ & $0.64 \pm 0.13^{*}$ & $0.63 \pm 0.04^{\#}$ \\
TDI-S' (average mm/s) & $2.80 \pm 0.45$ & $3.03 \pm 0.30$ & $2.81 \pm 0.32$ & $2.68 \pm 0.25$ \\
Mitral E (cm/s) & $74.5 \pm 6.17$ & $74.3 \pm 9,65$ & $79.5 \pm 15,23$ & $71.9 \pm 9,92$ \\
Mitral A (cm/s) & $60.0 \pm 17.9$ & $61.5 \pm 19.5$ & $66.1 \pm 22.7$ & $53.4 \pm 15.6$ \\
E/A & $1.34 \pm 0.37$ & $1.35 \pm 0.41$ & $1.38 \pm 0.62$ & $1.45 \pm 0.36$ \\
IVRT (ms) & $30.1 \pm 3.29$ & $29.1 \pm 4.50$ & $36.1 \pm 5.15^{*}$ & $38.0 \pm 3.74^{\#}$ \\
TDI-E'/TDI-A' & $0.96 \pm 0.27$ & $0.83 \pm 0.24$ & $0.95 \pm 0.28$ & $1.07 \pm 0.24$ \\
E/TDI-E' (average) & $24.0 \pm 4.12$ & $24.4 \pm 5.17$ & $20.6 \pm 5.13$ & $21.9 \pm 5.03$ \\
\hline
\end{tabular}
imaging (average between lateral and septal wall velocity); E/A: ratio between early (E)-to-late (A) diastolic mitral inflow; IVRT: isovolumic relaxation time; TDI-E': mitral annular early velocity by tissue Doppler imaging (TDI); TDI-A': mitral annular late velocity by TDI; average: average between lateral and septal wall velocity. ANOVA and Tukey or Dunn test; * p<0.05 vs WKY-SED; \# p<0.05 vs WKY-EX

Fig. 3. Basal papillary muscle functional data. A: Developed tension. B: Maximum rate of tension development $(+\mathrm{dT} / \mathrm{dt})$. C: Resting tension. WKY-SED: sedentary Wistar-Kyoto rats; WKY-EX: exercised Wistar-Kyoto rats; SHR-SED: sedentary spontaneously hypertensive rats; SHR-EX: exercised spontaneously hypertensive rats. ANOVA and Tukey test; mean \pm standard deviation; \# $\mathrm{p}<0.05$ vs WKY-EX; $\S$ $\mathrm{p}<0.05$ vs SHR-SED.

Figure 4 shows the percentage of variation $(\Delta)$ between final and initial echocardiographic parameters calculated as [(final value minus initial value)/initial value] X 100. SHR-SED presented higher $\Delta \mathrm{LV}$ diastolic diameter than WKY-SED and SHREX groups. $\triangle$ LV mass was higher in SHRSED than WKY-SED and $\Delta$ midwall fractional shortening was lower in both SHR than their respective controls. $\Delta$ relative wall thickness did not differ statistically between groups.

\section{Myocardial function}

Basal papillary muscle functional data are shown in Figure 3. There were no differences between normotensive groups. SHR-EX presented higher developed tension than SHR-SED and WKY-EX. Table 4 presents developed tension after different inotropic stimulation.

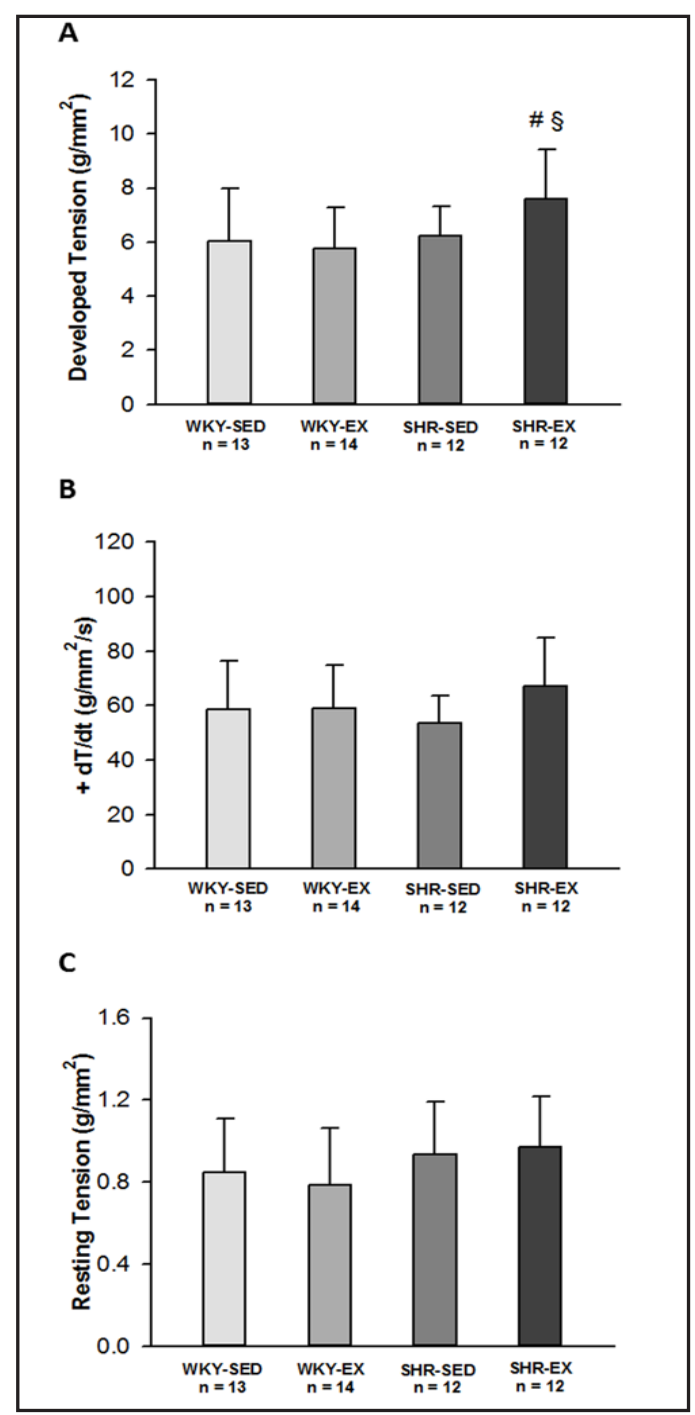




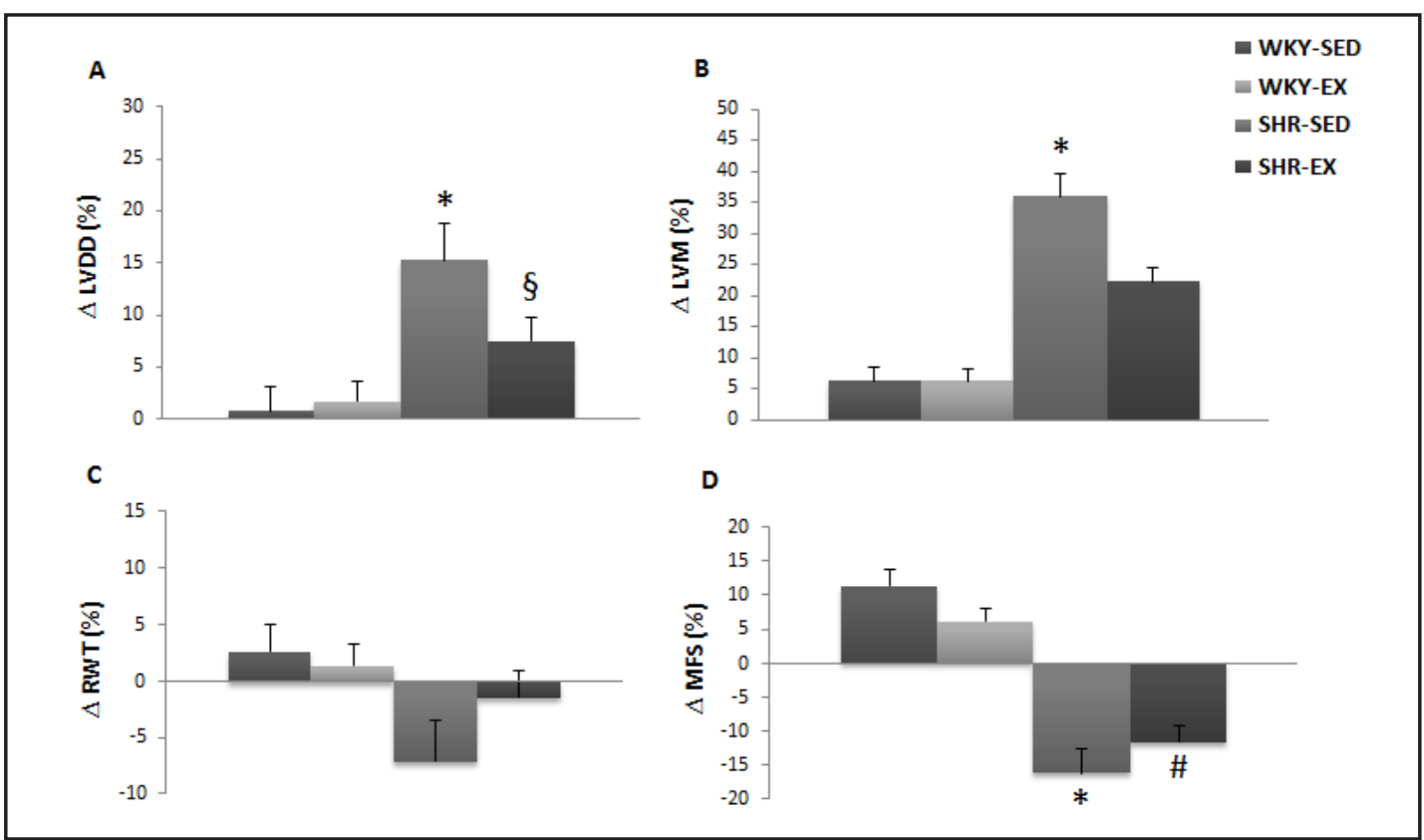

Fig. 4. Percentage of variation $(\Delta)$ between final and initial LV diastolic diameter (A), LV mass (B), LV relative wall thickness (C), and LV midwall fractional shortening (D) calculated as [(final minus initial value)/initial value] X 100. WKY-SED: sedentary Wistar-Kyoto rats; WKY-EX: exercised Wistar-Kyoto rats; SHR-SED: sedentary spontaneously hypertensive rats; SHR-EX: exercised spontaneously hypertensive rats. ANOVA and Tukey test; mean \pm standard deviation; * $\mathrm{p}<0.05$ vs WKY-SED; \# $\mathrm{p}<0.05$ vs WKY-EX; $§ \mathrm{p}<0.05$ vs SHR-SED.

Table 4. Developed tension (DT) after positive inotropic stimulation. Data as mean \pm standard deviation. WKY-SED: sedentary Wistar-Kyoto rats; WKY-EX: exercised Wistar-Kyoto rats; SHR-SED: sedentary spontaneously hyper-

\begin{tabular}{cccccc}
\hline & & $\begin{array}{c}\text { WKY-SED } \\
(\mathrm{n}=13)\end{array}$ & $\begin{array}{c}\text { WKY-EX } \\
(\mathrm{n}=14)\end{array}$ & $\begin{array}{c}\text { SHR-SED } \\
(\mathrm{n}=12)\end{array}$ & $\begin{array}{c}\text { SHR-EX } \\
(\mathrm{n}=12)\end{array}$ \\
\hline DT $\left(\mathrm{g} / \mathrm{mm}^{2}\right)$ & $10 \mathrm{~s}$ & $6.60 \pm 2.07$ & $6.33 \pm 1.63$ & $7.43 \pm 1.90$ & $8.30 \pm 2.01^{\#}$ \\
Post-resting contraction & $30 \mathrm{~s}$ & $6.97 \pm 2.25$ & $6.62 \pm 1.72$ & $7.76 \pm 2.04$ & $8.65 \pm 2.03^{\#}$ \\
& $60 \mathrm{~s}$ & $7.07 \pm 2.32$ & $6.72 \pm 1.75$ & $7.87 \pm 2.10$ & $8.74 \pm 2.09^{\#}$ \\
DT $\left(\mathrm{g} / \mathrm{mm}^{2}\right)$ & $0.625 \mathrm{mM}$ & $4.50 \pm 1.84$ & $4.49 \pm 1.50$ & $5.40 \pm 1.78$ & $6.19 \pm 1.61^{\#}$ \\
Extracellular $\left[\mathrm{Ca}^{2+}\right]$ & $1.25 \mathrm{mM}$ & $6.05 \pm 1.93$ & $5.77 \pm 1.53$ & $6.21 \pm 1.11$ & $7.60 \pm 1.83^{\#}$ \\
& $2.5 \mathrm{mM}$ & $6.62 \pm 2.17$ & $6.22 \pm 1.79$ & $7.12 \pm 2.22$ & $7.94 \pm 1.97$ \\
DT $\left(\mathrm{g} / \mathrm{mm}^{2}\right)$ & $10^{-8} \mathrm{M}$ & $5.58 \pm 2.04$ & $5.44 \pm 1.74$ & $6.41 \pm 2.15$ & $7.18 \pm 1.78^{\#}$ \\
Isoproterenol & $10^{-7} \mathrm{M}$ & $5.73 \pm 2.02$ & $5.40 \pm 1.74$ & $6.07 \pm 2.21$ & $6.88 \pm 2.12$ \\
& $10^{-6} \mathrm{M}$ & $5.85 \pm 2.07$ & $5.41 \pm 1.69$ & $5.87 \pm 2.05$ & $6.59 \pm 1.68$ \\
\hline
\end{tabular}
tensive rats; SHR-EX: exercised spontaneously hypertensive rats; DT: developed tension. ANOVA and Tukey test; ${ }^{*} \mathrm{p}<0.05$ vs WKY-SED; \# $\mathrm{p}<0.05$ vs WKY-EX; $§ \mathrm{p}<0.05$ vs SHR-SED

Table 5. Morphometric data. Data as mean \pm standard deviation. WKY-SED: sedentary Wistar-Kyoto rats; WKY-EX: exercised

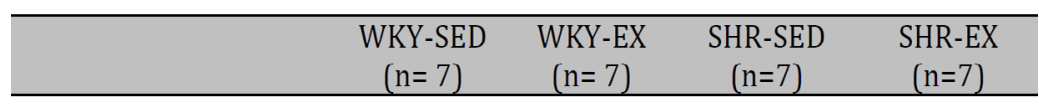

Myocyte diameter $(\mu \mathrm{m}) \quad 19.7 \pm 0.69 \quad 20.4 \pm 1.31 \quad 22.3 \pm 2.38^{*} \quad 21.1 \pm 1.62$

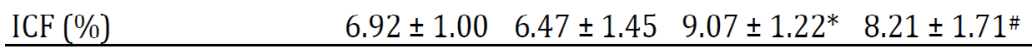
Wistar-Kyoto rats; SHR-SED: sedentary spontaneously hypertensive rats; SHR-EX: exercised spontaneously hypertensive rats; ICF: interstitial collagen fraction. ANOVA and Tukey test; * p <0.05 vs WKY-SED; \# p<0.05 vs WKY-EX

\section{Morphologic evaluation}

Left ventricular myocyte diameter was higher in SHR-SED than WKY-SED (Table 5). Interstitial collagen fraction was higher in hypertensive than normotensive groups. 
Fig. 5. Left ventricular myocardial hydroxyproline concentration (HOP). WKY-SED: sedentary Wistar-Kyoto rats; WKY-EX: exercised Wistar-Kyoto rats; SHR-SED: sedentary spontaneously hypertensive rats; SHR-EX: exercised spontaneously hypertensive rats. ANOVA and Tukey test; mean \pm standard deviation; ${ }^{*} \mathrm{p}<0.05$ vs WKY-SED; \# p<0.05 vs WKY-EX; $§ \mathrm{p}<0.05$ vs SHR-SED.

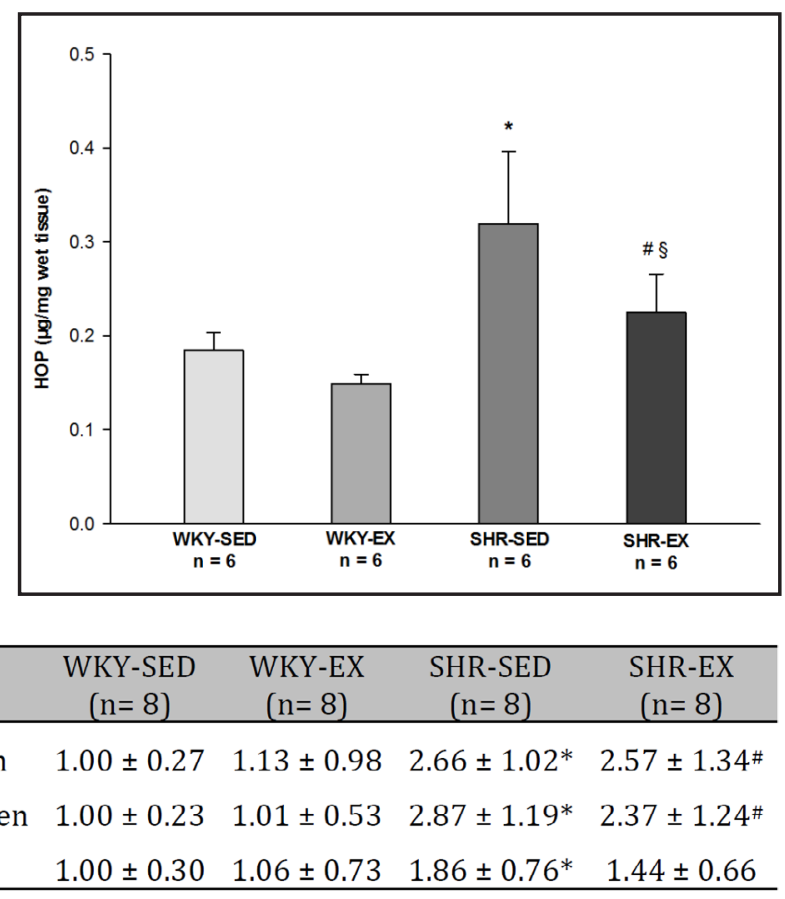

Table 6. Collagen and lysyl oxidase gene expression Data as mean \pm standard deviation. WKY-SED: sedentary WistarKyoto rats; WKY-EX: exercised Wistar-Kyoto rats; SHR-SED:

\begin{tabular}{lcccc}
\hline & $\begin{array}{c}\text { WKY-SED } \\
(\mathrm{n}=8)\end{array}$ & $\begin{array}{c}\text { WKY-EX } \\
(\mathrm{n}=8)\end{array}$ & $\begin{array}{c}\text { SHR-SED } \\
(\mathrm{n}=8)\end{array}$ & $\begin{array}{c}\text { SHR-EX } \\
(\mathrm{n}=8)\end{array}$ \\
\hline Type I collagen & $1.00 \pm 0.27$ & $1.13 \pm 0.98$ & $2.66 \pm 1.02^{*}$ & $2.57 \pm 1.34^{\#}$ \\
Type III collagen & $1.00 \pm 0.23$ & $1.01 \pm 0.53$ & $2.87 \pm 1.19^{*}$ & $2.37 \pm 1.24^{\#}$ \\
Lysyl oxidase & $1.00 \pm 0.30$ & $1.06 \pm 0.73$ & $1.86 \pm 0.76^{*}$ & $1.44 \pm 0.66$ \\
\hline
\end{tabular}
sedentary spontaneously hypertensive rats; SHR-EX: exercised spontaneously hypertensive rats. ANOVA and Tukey test; * ${ }^{*}<0.05$ vs WKY-SED; \# p<0.05 vs WKY-EX

\section{Collagen assessment}

Left ventricular myocardial hydroxyproline concentration was higher in both SHR groups than WKY and lower in SHR-EX than SHR-SED (Fig. 5). Collagen type I and III gene expression was higher in SHR than WKY groups. Lysyl oxidase gene expression was higher in SHR-SED than WKY-SED; in SHR-EX, this parameter did not differ from either WKY-EX or SHR-SED (Table 6).

\section{Discussion}

In this study, we showed that aging spontaneously hypertensive rats subjected to a long-term low intensity aerobic exercise protocol present improved functional capacity and a reduced frequency of decompensated heart failure. Improved clinical status was combined with enhanced left ventricular and myocardial function and reduced myocardial interstitial collagen.

SHR have been widely used to study cardiac remodeling and the transition from compensated left ventricular hypertrophy to decompensated heart failure. These rats were introduced by Okamoto and Aoki [46] as a genetic model of hypertension very similar to hypertension in humans. After developing early arterial hypertension and left ventricular hypertrophy, SHR remain compensated for a long period [23, 47]. From 18 months of age, SHR start to present clinical and pathological heart failure features such as tachypnea, ascites, pleural effusion, atrial thrombus, lung congestion, and right ventricular hypertrophy $[24,48]$. Left untreated, rats evolve to death within two to four weeks. We therefore initiated the exercise protocol at age 16 months when no SHR had tachypnea.

We used a low intensity protocol of aerobic exercise, adapted from published studies on aged untreated SHR $[30,49]$. Younger untreated SHR (11 months) had experienced sudden death at a running speed of $17.5 \mathrm{~m} / \mathrm{min}$ [30]. We therefore subjected our rats to physical exercise at $12 \mathrm{~m} / \mathrm{min}$, a tolerable intensity for all rats. This protocol proved efficient as functional capacity was increased in exercised compared to sedentary groups. In both the 
beginning and end periods of the exercise protocol, SHR presented better functional capacity than WKY rats. Previous studies have shown that SHR are more physically active, therefore presenting better functional capacity than normotensive rats [50, 51]. Improved functional capacity in SHR occurred despite a slight increase in arterial blood pressure compared to SHR-SED. The effects of exercise on SHR blood pressure are variable; some authors have shown an anti-hypertensive effect of exercise [19], others found hypertension unchanged $[52,53]$ or even exacerbated [54]. The low intensity exercise protocol did not change body weight. As usually seen in literature, our SHR presented lower body weight than WKY groups $[23,24]$.

At euthanasia, SHR-SED showed a significantly higher frequency of tachypnea, pleural effusion, and ascites than the WKY-SED group. In SHR-EX, the frequency of tachypnea, pleural effusion, and ascites was lower than SHR-SED and did not significantly differ from WKY-SED. This shows that even in the face of slightly increased arterial hypertension, low-intensity exercise training was capable of reducing the occurrence of decompensated heart failure in untreated SHR. Similar results have previously been observed in male [30] and female [49] aged SHR.

We next evaluated whether improved ventricular and myocardial function was involved in better functional capacity and a reduction in decompensated heart failure frequency.

Cardiac structures and LV function were analyzed by conventional transthoracic echocardiogram and tissue Doppler imaging, and myocardial function was evaluated in LV papillary muscle preparations. Exercise in WKY rats did not change any cardiac parameters showing that increased functional capacity is not related to improved cardiac performance in normotensive rats. SHR-SED presented concentric left ventricular hypertrophy, characterized by increased LV posterior and septal wall thickness, relative wall thickness and LV mass, with systolic dysfunction, verified by decreased endocardial and midwall fractional shortening and posterior wall shortening velocity, and increased Tei index. Concerning diastolic function, only isovolumetric relaxation index was changed. Myocardial function did not differ from WKY-SED group. Therefore SHR-SED presented impaired in vivo systolic ventricular function with unchanged in vitro myocardial function. SHR usually present enhanced myocardial performance up to 18 months of age compared to WKY rats. As they age, SHR present unchanged function, and finally a depressed myocardial function $[6,23]$. Thus, impaired in vivo ventricular function probably reflects the effect of increased afterload on LV systolic function.

To the best of our knowledge, this is the first study to evaluate the effects of exercise in aging SHR using tissue Doppler imaging and papillary muscle preparations. In echocardiographic assessment, no parameters differed between SHR-EX and SHR-SED groups. However, the percentage of variation between final and initial LV diastolic diameter was lower in SHR-EX than SHR-SED, showing that exercise attenuated LV dilation in SHR. Furthermore, SHR-EX LV systolic diameter, which was higher in SHR-SED than WKY-SED, and endocardial fractional shortening, which was lower in SHR-SED than WKY-SED, had values between WKY-EX and SHR-SED and did not differ statistically from either of these groups, suggesting LV dysfunction was attenuated by exercise.

Papillary muscle preparations allow the evaluation of myocardial function without influence from cardiac load and ventricular chamber geometry which can alter in vivo cardiac performance. At baseline, SHR-EX presented higher developed tension than WKY-EX and SHR-SED. Positive inotropic stimulation has been used to evaluate myocardial contractile reserve and to identify contraction and relaxation changes that are not observed under basal conditions [39]. After inotropic stimulation, SHR-SED did not differ from WKY-SED. However SHR-EX, although not differing from SHR-SED, presented higher developed tension than WKY-EX after post-rest contractions, in contractions with 0.625 and $1.25 \mathrm{mM}$ extracellular calcium concentrations, and after the addition of $10^{-8} \mathrm{M}$ isoproterenol to nutrient solution. Thus echocardiogram and papillary muscle preparation results suggest that exercise attenuated myocardial function deterioration thus improving functional capacity and reducing the occurrence of decompensated heart failure. 


\section{Cellular Physiology Cell Physiol Biochem 2015;36:61-74 \begin{tabular}{l|l} 
and Biochemistry Publisned onIIne: April 27, 2015 & $\begin{array}{l}\text { C) 2015 S. Karger AG, Basel } \\
\text { www.karger.com/cpb }\end{array}$ \\
\hline
\end{tabular}}

Pagan et al.: Exercise in Aging Spontaneously Hypertensive Rats

Long-term pressure overload-induced cardiac remodeling mainly consists of cardiomyocyte hypertrophy and changes in phenotype and the amount of myocardial collagen. We therefore evaluated the degree of myocyte hypertrophy and myocardial fibrosis. SHR-SED had higher values of myocyte small diameter than WKY-SED. Despite unchanged left ventricle weight, SHR-EX myocyte diameter was between those of WKY-EX and SHR-SED and did not significantly differ from either group suggesting that hypertrophy was attenuated by exercise, independent of the elevated systemic arterial pressure. In contrast, other authors have observed increased SHR myocyte hypertrophy after exercise. The different response can be attributed to differences in exercise intensity [28], or animal gender [30] and age [14].

In this study, myocardial fibrosis was evaluated by myocardial morphometry, biochemical analysis, and gene expression. The interstitial collagen fraction increased in both SHR groups compared to WKY and was unchanged by physical exercise. However, myocardial hydroxyproline concentration was reduced in SHR-EX compared to SHR-SED. Hydroxyproline, an amino acid, is the main component of the collagen molecule and can only be found in small concentrations in a limited number of other proteins. Evaluation of myocardial fibrosis using hydroxyproline is more precise than histological analyses [55]. Cardiac mechanical properties are not only modulated by the amount of myocardial collagen but also by the type of collagen in the myocardium. Fibrillar collagen types I and III are the predominant components of the cardiac extracellular matrix [56]. Tissue predominantly containing type I collagen is stiffer than tissue composed of higher type III fiber concentrations $[57,58]$. In this study, both type I and type III collagen gene expression were increased in SHR groups and unchanged by exercise. Gene expression of lysyl oxidase, an enzyme important in collagen cross-link formation, was higher in SHR-SED than WKYSED; it did not differ from the other groups in SHR-EX. We therefore conclude that long-term physical exercise attenuated increase in myocardial collagen content and lysyl oxidase gene expression. It has previously been shown that exercise training in both normotensive and hypertensive aging rats attenuates myocardial fibrosis and modulates myocardial collagen gene expression [19, 59].

Studies on aging SHR have shown that heart failure development is associated with marked myocardial fibrosis and impaired contractile function, which suggests that fibrosis or changes in connective tissue response are important during the transition from compensated hypertrophy to failure [6]. Therefore myocardial fibrosis, by restricting myofibrillar motion [6], may have contributed to the impaired cardiac function. Thus, in this study, exercise-induced attenuation of myocyte hypertrophy and fibrosis is probably involved in improved functional capacity and myocardial and cardiac function and a reduced frequency in heart failure development. Additional studies are necessary to evaluate other potential mechanisms underlying the beneficial effects induced by exercise.

This study has important clinical implications as geriatric patients are usually resistant to exercise programs specially if exercise intensity is high. Therefore, our results suggest that an aging population with chronic pressure overload can gain beneficial effects in clinical status from low intensity aerobic physical exercise.

In conclusion, a long-term low intensity physical exercise protocol improves functional capacity and reduces the frequency of decompensated heart failure in aging spontaneously hypertensive rats independent of elevated systemic arterial pressure. Improvement in functional status is combined with attenuation in left ventricular and myocardial dysfunction and myocardial fibrosis.

\section{Acknowledgements}

We are grateful to Jose Carlos Georgette and Mario Batista Bruno for their technical assistance and Colin Edward Knaggs for English editing.

Financial support was provided by CNPq (306857/2012-0, 306845/2012-1, and 480829/2013-6), FAPESP (2009/54506-7), CAPES, and PROPe, UNESP. 


\section{Cellular Physiology and Biochemistry}

Cell Physiol Biochem 2015;36:61-74

DOI: 10.1159/000374053

Published onIIne: April 27, 2015

(C) 2015 S. Karger AG, Base

Pagan et al.: Exercise in Aging Spontaneously Hypertensive Rats

\section{Disclosures Statement}

The authors report no conflict of interest.

\section{References}

1 Egan BM, Li J, Shatat IF, Fuller JM, Sinopoli A: Closing the gap in hypertension control between younger and older adults: National Health and Nutrition Examination Survey (NHANES) 1988 to 2010. Circulation 2014;129:2052-2061.

-2 Diwan A, Dorn GW 2nd: Decompensation of cardiac hypertrophy: cellular mechanisms and novel therapeutic targets. Physiology 2007;22:56-64.

3 Drazner MH: The progression of hypertensive heart disease. Circulation 2011;123:327-334.

-4 Laroumanie F, Douin-Echinard V, Pozzo J, Lairez O, Tortosa F, Vinel C, Delage C, Calise D, Dutaur M, Parini A, Pizzinat N: CD4+ T cells promote the transition from hypertrophy to heart failure during chronic pressure overload. Circulation 2014;129:2111-2124.

-5 Opie LH, Commerford PJ, Gersh BJ, Pfeffer MA: Controversies in ventricular remodelling. Lancet 2006;367:356-367.

6 Conrad CH, Brooks WW, Hayes JA, Sen S, Robinson KG, Bing OHL: Myocardial fibrosis and stiffness with hypertrophy and heart failure in the spontaneously hypertensive rat. Circulation 1995;91:161-170.

7 Moore-Morris T, Guimarães-Camboa N, Banerjee I, Zambon AC, Kisseleva T, Velayoudon A, Stallcup WB, Gu Y, Dalton ND, Cedenilla M, Gomez-Amaro R, Zhou B, Brenner DA, Peterson KL, Chen J, Evans SM: Resident fibroblast lineages mediate pressure overload-induced cardiac fibrosis. J Clin Invest 2014;124:2921-2934. Mancia G, Fagard R, Narkiewicz K, Redon J, Zanchetti A, Bohm M, Christiaens T, Cifkova R, Backer G, Dominiczak A, Galderisi M, Grobbee DE, Jaarsma T, Kirchhof P, Kjeldsen SE, Laurent S, Manolis AJ, Nilsson PM, Ruilope LM, Schmieder RE, Sirnes PA, Sleight P, Viigimaa M, Waeber B, Zannad F: 2013 ESH/ESC guidelines for the management of arterial hypertension. The Task Force for the management of arterial hypertension of the European Society of Hypertension (ESH) and of the European Society of Cardiology (ESC). J Hypertens 2013;31:1281-1357.

-9 Yancy CW, Jessup M, Bozkurt B, Butler J, Casey Jr. DE, Drazner MH, Fonarow GC, Geraci SA, Horwich T, Januzzi JL, Johnson MR, Kasper EK, Levy WC, Masoudi FA, McBride PE, McMurray JJ, Mitchell JE, Peterson PN, Riegel B, Sam F, Stevenson LW, Tang WH, Tsai EJ, Wilkoff BL: 2013 ACCF/AHA guideline for the management of heart failure: executive summary: a report of the American College of Cardiology Foundation/American Heart Association Task Force on practice guidelines. Circulation 2013;128:18101852.

10 Flynn KE, Pina IL, Whellan DJ, Lin L, Blumenthal JA, Ellis SJ, Fine LJ, Howlett JG, Keteyian SJ, Kitzman DW, Kraus WE, Miller NH, Schulman KA, Spertus JA, O'Connor CM, Weinfurt KP: Effects of exercise training on health status in patients with chronic heart failure. J Am Med Assoc 2009;301:1451-1459.

11 Giannuzzi P, Temporelli PL, Corra U, Tavazzi L, Group E-CS: Antiremodeling effect of long-term exercise training in patients with stable chronic heart failure: results of the exercise in left ventricular dysfunction and chronic heart failure (ELVD-CHF) trial. Circulation 2003;108:554-559.

12 Nishi I, Noguchi T, Iwanaga Y, Furuichi S, Aihara N, Takaki H, Goto I: Effects of exercise training in patients with chronic heart failure and advanced left ventricular systolic dysfunction receiving $\beta$-blockers. Circ J 2011;75:1649-1655.

13 Wisloff U, Stoylen A, Loennechen JP, Bruvold M, Rognmo O, Haram PM, Tjonna AE, Helgerud J, Slordahl SA, Lee SJ, Videm V, Bye A, Smith GL, Najjar SM, Ellingsen O, Skjaerpe T: Superior cardiovascular effect of aerobic interval training versus moderate continuous training in heart failure patients. A randomized study. Circulation 2007;115:3086-3094.

14 Garciarena CD, Pinilla OA, Nolly MB, Laguens RP, Escudero EM, Cingolani HE, Ennis IL: Endurance training in the spontaneously hypertensive rat. Conversion of pathological into physiological cardiac hypertrophy. Hypertension 2009;53:708-714.

15 Kraljevic J, Marinovic J, Pravdic D, Zubin P, Dujic Z, Wisloff U, Ljubkovic M: Aerobic interval training attenuates remodelling and mitochondrial dysfunction in the post-infarction failing rat heart. Cardiovasc Res 2013;99:55-64.

16 Llewellyn TL, Sharma NM, Zheng H, Patel KP: Effects of exercise training on sfo-mediated sympathoexcitation during chronic heart failure. Am J Physiol Heart Circ Physiol 2014;306:H121-H131.

17 Medeiros A, Rolim NP, Oliveira RS, Rosa KT, Mattos KC, Casarini DE, Irigoyen MC, Krieger EM, Krieger JE, Negrao CE, Brum PC: Exercise training delays cardiac dysfunction and prevents calcium handling abnormalities in sympathetic hyperactivity-induced heart failure mice. J Appl Physiol 2008;104:103-109. 


\section{Cellular Physiology Cell Physiol Biochem 2015;36:61-74 \begin{tabular}{l|l|l}
\hline DOI: 10.1159/000374053 & (C) 2015 S. Karger AG, Basel
\end{tabular}

Pagan et al.: Exercise in Aging Spontaneously Hypertensive Rats

18 Nunes RB, Alves JP, Kessler LP, Dal Lago P: Aerobic exercise improves the inflammatory profile correlated with cardiac remodeling and function in chronic heart failure rats. Clinics 2013;68:876-882.

19 Rossoni LV, Oliveira RA, Caffaro RR, Miana M, Sanz-Rosa D, Koike MK, Do Amaral SL, Michelini LC, Lahera V, Cachofeiro V: Cardiac benefits of exercise training in aging spontaneously hypertensive rats. J Hypertens 2011;29:2349-2358.

20 Xu X, Wan W, Powers AS, Li J, Ji LL, Lao S, Wilson B, Erikson JM, Zhang JQ: Effects of exercise training on cardiac function and myocardial remodeling in post myocardial infarction rats. J Mol Cell Cardiol 2008;44:114-122.

21 Villella M, Villella A: Exercise and cardiovascular diseases. Kidney Blood Press Res 2014;39:147-153.

22 Melo SF, Fernandes T, Baraúna VG, Matos KC, Santos AA, Tucci PJ, Oliveira EM: Expression of microRNA-29 and collagen in cardiac muscle after swimming training in myocardial-infarcted rats. Cell Physiol Biochem 2014;33:657-669.

-23 Cicogna AC, Robinson KG, Conrad CH, Singh K, Squire R, Okoshi MP, Bing OHL: Direct effects of colchicine on myocardial function. Studies in hypertrophied and failing spontaneously hypertensive rats. Hypertension 1999;33:60-65.

24 Damatto RL, Martinez PF, Lima AR, Cezar MD, Campos DH, Oliveira SAJ, Guizoni DM, Bonomo C, Nakatani BT, Dal Pai Silva M, Carvalho RF, Okoshi K, Okoshi MP: Heart failure-induced skeletal myopathy in spontaneously hypertensive rats. Int J Cardiol 2013;167:698-703.

25 Cicogna AC, Padovani CR, Okoshi K, Aragon FF, Okoshi MP: Myocardial function during chronic food restriction in isolated hypertrophied cardiac muscle. Am J Med Sci 2000;320:244-248.

-26 Okoshi MP, Okoshi K, Matsubara LS, Pai-Silva MD, Gut AL, Padovani CR, Pai VD, Cicogna AC: Myocardial remodeling and dysfunction are induced by chronic food restriction in spontaneously hypertensive rats. Nutr Res 2006;26:567-572.

-27 Cezar MD, Damatto RL, Martinez PF, Lima AR, Campos DH, Rosa CM, Guizoni DM, Bonomo C, Cicogna AC, Gimenes R, Pagan LU, Okoshi MP, Okoshi K: Aldosterone blockade reduces mortality without changing cardiac remodeling in spontaneously hypertensive rats. Cell Physiol Biochem 2013;32:1275-1287.

28 Schultz RL, Swllow JG, Waters RP, Kuzman JA, Redetzke RA, Said S, Escobar GM, Gerdes AM: Effects of excessive long-term exercise on cardiac function and myocyte remodeling in hypertensive heart failure rats. Hypertension 2007;50:410-416.

-29 Schultz RL, Kullman EL, Waters RP, Huang H, Kirwan JP, Gerdes AM, Swallow JG: Metabolic adaptations of skeletal muscle to voluntary wheel running exercise in hypertensive heart failure rats. Physiol Res 2013;62:361-369.

-30 Emter CA, McCune SA, Sparagna GC, Radin MJ, Moore RL: Low-intensity training delays onset of decompensated heart failure in spontaneously hypertensive heart failure rats. Am J Physiol Heart Circ Physiol 2005;289:H2030-H2038.

31 Gimenes C, Gimenes R, Rosa CM, Xavier NP, Campos DHS, Fernandes AAH, Cezar MDM, Guirado GN, Cicogna AC, Takamoto AHR, Okoshi MP, Okoshi K: Low intensity physical exercise attenuates cardiac remodeling and myocardial oxidative stress and dysfunction in diabetic rats. J Diabetes Res 2015, in press.

-32 Lima AR, Martinez PF, Okoshi K, Guizoni DM, Zornoff LA, Campos DH, Oliveira SAJ, Bonomo C, Pai-Silva MD, Okoshi MP: Myostatin and follistatin expression in skeletal muscles of rats with chronic heart failure. Int J Exp Path 2010;91:54-62.

-33 Martinez PF, Okoshi K, Zornoff LA, Oliveira SAJ, Campos DH, Lima AR, Damatto RL, Cezar MD, Bonomo C, Guizoni DM, Padovani CR, Cicogna AC, Okoshi MP: Echocardiographic detection of congestive heart failure in postinfarction rats. J Appl Physiol 2011;111:543-551.

34 Okoshi K, Ribeiro HB, Okoshi MP, Matsubara BB, Gonçalves G, Barros R, Cicogna AC: Improved systolic ventricular function with normal myocardial mechanics in compensated cardiac hypertrophy. Jpn Heart J 2004;45:647-656.

-35 de Paiva SAR, Zornoff LAM, Okoshi MP, Okoshi K, Matsubara LS, Matsubara BB, Cicogna AC, Campana AO: Ventricular remodeling induced by retinoic acid supplementation in adult rats. Am J Physiol Heart Circ Physiol 2003;284:H2242-H2246.

-36 Sugizaki MM, Carvalho RF, Aragon FF, Padovani CR, Okoshi K, Okoshi MP, Zanati SG, Pai-Silva MD, Novelli EL, Cicogna AC: Myocardial dysfunction induced by food restriction is related to morphological damage in normotensive middle-aged rats. J Biomed Sci 2005;12:641-649.

37 Lang RM, Bierig M, Devereux RB, Flachskampf FA, Foster E, Pellikka PA, Picard MH, Roman MJ, Seward J, Shanewise JS, Solomon SD, Spencer KT, Sutton MS, Stewart WJ: Recommendations for chamber quantification: a report from the American Society of Echocardiography's Guidelines and Standards Committee and the Chamber Quantification Writing Group, developed in conjunction with the European Association of Echocardiography, a branch of the European Society of Cardiology. J Am Soc Echocardiogr 2005;18:1440-1463. 


\section{Cellular Physiology Cell Physiol Biochem 2015;36:61-74 \begin{tabular}{l|l|l}
\hline DOI: 10.1159/000374053 & (C) 2015 S. Karger AG, Basel
\end{tabular}

Pagan et al.: Exercise in Aging Spontaneously Hypertensive Rats

38 Cicogna AC, Padovani CR, Okoshi K, Matsubara LS, Aragon FF, Okoshi MP: The influence of temporal food restriction on the performance of isolated cardiac muscle. Nutr Res 2001;21:639-648.

-39 Gut AL, Okoshi MP, Padovani CR, Aragon FF, Cicogna AC: Myocardial dysfunction induced by food restriction is related to calcium cycling and beta-adrenergic system changes. Nutr Res 2003;23:911-919.

40 Okoshi MP, Okoshi K, Pai VD, Pai-Silva MD, Matsubara LS, Cicogna AC: Mechanical, biochemical, and morphological changes in the heart from chronic food restricted rats. Can J Physiol Pharmacol 2001;79:754-760.

41 Rosa CM, Xavier NP, Campos DH, Fernandes AA, Cezar MD, Martinez PF, Cicogna AC, Gimenes C, Gimenes R, Okoshi MP, Okoshi K: Diabetes mellitus activated fetal gene program and intensifies cardiac remodeling and oxidative stress in aged spontaneously hypertensive rats. Cardiovasc Diabetol 2013;12:152.

42 Oliveira Junior SA, Dal Pai-Silva M, Martinez PF, Lima-Leopoldo AP, Campos DH, Leopoldo AS, Okoshi MP, Okoshi K, Padovani CR, Cicogna AC: Diet-induced obesity causes metabolic, endocrine and cardiac alterations in spontaneously hypertensive rats. Med Sci Monit 2010;16:BR367-BR373.

43 Lima ARR, Martinez PF, Damatto RL, Cezar MDM, Guizoni DM, Bonomo C, Oliveira SAJ, Dal-Pai Silva M, Zornoff LAM, Okoshi K, Okoshi MP: Heart failure-induced diaphragm myopathy. Cell Physiol Biochem 2014;34:333-345.

44 Martinez PF, Okoshi K, Zornoff LA, Carvalho RF, Oliveira Junior SA, Lima AR, Campos DH, Damatto RL, Nogueira CR, Dal Pai-Silva M, Okoshi MP: Chronic heart failure-induced skeletal muscle atrophy, necrosis, and myogenic regulatory factors changes. Med Sci Monit 2010;16:374-383.

-45 Martinez PF, Bonomo C, Guizoni DM, Oliveira Junior AS, Damatto RL, Cezar MDM, Lima ARR, Pagan LU, Seiva FR, Fernandes DC, Laurindo FRM, Novelli ELB, Matsubara LS, Zornoff LAM, Okoshi K, Okoshi MP: Influence of $\mathrm{N}$-acetylcysteine on oxidative stress in slow-twitch soleus muscle of heart failure rats. Cell Physiol Biochem 2015;35:148-159.

46 Okamoto K, Aoki K: Development of a strain of spontaneously hypertensive rats. Jpn Circ J 1963;27:282293.

-47 Bing OHL, Brooks WW, Robinson KG, Slawsky MT, Hayes JA, Litwin SE, Sen S, Conrad CH: The spontaneously hypertensive rat as a model of the transition from compensated left ventricular hypertrophy to failure. J Mol Cell Cardiol 1995;27:383-396.

48 Boluyt MO, Bing OHL, Lakatta EG: The ageing spontaneously hypertensive rat as a model of the transition from stable compensated hypertrophy to heart failure. Eur Heart J 1995;16:19-30.

49 Chicco AJ, McCune SA, Emter CA, Sparagna GC, Rees ML, Bolden DA, Marshall KD, Murphy RC, Moore RL: Low-intensity exercise training delays heart failure and improves survival in female hypertensive heart failure rats. Hypertension 2008;51:1096-1102.

50 Robinson AM, Hopkins ME, Bucci DJ: Effects of physical exercise on ADHD-like behavior in male and female adolescent spontaneously hypertensive rats. Dev Psychobiol 2011;53:383-390.

51 Langen B, Dost R: Comparison of SHR, WKY and Wistar rats in different behavioural animal models: effect of dopamine D1 and alpha2 agonists. Atten Defic Hyperact Disord 2011;3:1-12.

52 Renna BF, MacDonnell SM, Reger PO, Crabbe DL, Houser SR, Libonati JR: Relative systolic dysfunction in female spontaneously hypertensive rat myocardium. J Appl Physiol 2007;103:353-358.

53 Tomanek RJ, Gisolfi CV, Bauer CA, Palmer PJ: Coronary vasodilator reserve, capillarity, and mitochondria in trained hypertensive rats. J Appl Physiol 1988;64:1179-1185.

54 Golden AL, Bright JM, Lawler JE: Changes in creatine kinase expression induced by exercise in borderline hypertensive rat hearts. Clin Exp Hypertens 1994;16:577-593.

55 Matsubara LS, Bojikian BB, Okoshi MP, Cicogna AC, Janicki JS: Alterations in myocardial collagen content affect rat papillary muscle function. Am J Physiol 2000;279:H1534-H1539.

56 Fan D, Takawale A, Lee J, Kassiri Z: Cardiac fibroblasts, fibrosis and extracellular matrix remodeling in heart disease. Fibrogenesis Tissue Repair 2012;5:5-15.

57 Brower GL, Gardner JD, Forman MF, Murray DB, Voloshenyuk T, Levick SP, Janicki JS: The relationship between myocardial extracellular matrix remodeling and ventricular function. Eur J Cardiothorac Surg 2006;30:604-610.

58 Lopez B, Gonzalez A, Hermida N, Valencia F, de Teresa E, Diez J: Role of lysyl oxidase in myocardial fibrosis: From basic science to clinical aspects. Am J Physiol Heart Circ Physiol 2010;299:H1-H9.

59 Thomas DP, Zimmerman SD, Hansen TR, Martin DT, McCormick RJ: Collagen gene expression in rat left ventricle: interactive effect of age and exercise training. J Appl Physiol 2000;89:1462-1468. 